25 cc $n / 20$ alkoholische Kalilauge dazugegeben und das Kölbchen mit einem langen Luftkuhler verbunden wurde. Dann wurde es 10 Minuten bzw. 1/2 Stunde in das siedende Wasserbad gebracht und hierauf zurticktitriert. Die folgenden Zablen wurden erhalten

\begin{tabular}{|c|c|c|c|c|}
\hline \multirow{8}{*}{ 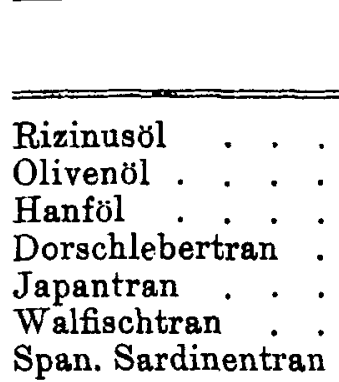 } & \multicolumn{2}{|c|}{$\begin{array}{l}\text { Nach } 10 \\
\text { Minuten }\end{array}$} & \multicolumn{2}{|c|}{$\begin{array}{c}\text { Nach } \\
1 / 2 \text { Stunde }\end{array}$} \\
\hline & 181,0 & 182,0 & 181,8 & 181,9 \\
\hline & 167,0 & 175,8 & 207,4 & 208,0 \\
\hline & 186,2 & 162,6 & 193,0 & 192,5 \\
\hline & 175,0 & 172,0 & 176,5 & 175,5 \\
\hline & 179,0 & 186,3 & 183,5 & 185,0 \\
\hline & 166,5 & 139,0 & 188,3 & - \\
\hline & 101,6 & 76,5 & 124,1 & 126,3 \\
\hline
\end{tabular}

Zu den Angaben Woodroffes wäre nun folgendes zu bemerken.

25 cc $n / 10$ alkoholische Kalilauge enthalten, wie leicht $z \mathrm{u}$ berechnen, $140 \mathrm{mg} \mathrm{KOH}$. $2 \mathrm{~g}$ Oel dagegen erfordern bei einer durchschnittlichen Verseifungszahl von 190 mindestens $380 \mathrm{mg} \mathrm{KOH}$, ungerechnet den notwendigen Ueberschuss. Das Referat muss also einen Druckfehler enthalten, es muss ohne $\mathrm{Z}$ weifel anstatt $\mathrm{n} / \mathrm{10}^{\mathrm{n}} \mathrm{n} / \mathrm{2}$ alkoholische Kalilauge heissen.

$\mathrm{Ob}$ W. dem Vorschlag 2 gerecht wurde, d. h. ob seine Lauge weniger als $10 \%$ Wasser enthielt, geht aus dem Referat nicht hervor, es ist aber sehr wesentlich, denn ein höherer Wassergehalt verzögert dieVerseitung. Dass der Bedingung 4 Gentige geleistet wurde, ist anzunehmen, trotzdem bei blosser Luftkuhlung in $1 / 2$ Stunde ziemlich viel Alkohol verloren gehen kann.

Nur beim Rizinusöl gentugte 10 Minuten langes Erhitzen in beiden Fällen. Das stimmt mit der bekannten Tatsache uberein, dass die Glyzeride von Oxyfettsäuren leichter verseifbar sind als diejenigen normaler Fettsäuren. Beim Dorschleber- und beim Japantran gentigten 10 Minuten wenigstens in einem von zwei Fällen, in allen anderen Fällen war ein nur 10 Minuten währendes Erhitzen zu wenig.

Trotzdem kann ich W. auf das Bestimmteste versichern, dass 10 Minuten langes Kochen unter allen Unständen gentigt, wenn man nach meiner Methode verführt und in dieser Beziehung muss ich zugeben, dass ich mich vielleicht nicht genau genug ausgedrtickt habe. Ich "erhitze" nämlich nicht auf dem Wasserbad, sondern ich "koche" in einem Erlenmeyerkolben direkt auf dem Drahtnetz unter öfterem Umschwenken, das die Verseifung sehr beschleunigt. Man hat so vor allen Dingen den schon fruher erwähnten Vorteil, dass man den Verseifungsprozess mit dem Auge verfolgen kann und sieht, wie die Oeltröpfchen allmählich verschwinden und eino rollkommen blanke Seifenlösung entsteht. Eine Kuhlung ist uberflussig, wenn man den verdunsteten Alkohol vor der Titration wieder ersetzt. Ich habe Hunderte von Bestimmungen in dieser Weise ausgefuhrt, vor allen Dingen auch diejenigen in der zweiten Tabelle, und W. wird nicht behaupten wollen, dass auch nur eine der 8 Zahlen zu niedrig ist.

Im einzelnen wäre vielleicht noch anzufügen, dass das Olivenöl Woodroffes eine besondere Qualität sein muss, denn nach meinen Erfahrungen liegt die Verseifungszahl normalen Olivenöls unter 200. Der spanische Sardinentran war entweder auch nach $1 / a$ Stunde noch nicht vollständig verseift oder er war mit Mineralöl verschnitten, denn einen reinen Sardinentran mit der Verseifungszahl 126 gibt es nicht.

\title{
Aus der Technik der Raffination des Erdöls.
}

Von Dr. Richard Kisgling.

(Fortsetzung.)

1. Die Verarbeitung einer pennsylvanischen Erdölsorte.

a) Zur Gewinnung ron Benzin, Leuchtõ, Mittelöl, Lagerschmieröl und Paraffin.

Vom Rohöl, das auf die oben angegebeno Weise vom Wasser befreit ist, destilliert man zunächst mittels Dampfheizung die niedrigsiedenden, auf Benzin zu verarbeitenden Anteile ab und unterstutzt zuletzt die Destil- lation durch Einfuhrung eines Dampfstromes in das Oel. Wenn das spez. Gew. des aus dem Kuhler tretenden Destillates 0.730 bis 0.735 beträgt, unterbricht man die Destillation. Das entbenzinierte Oel wird nach seiner $\mathrm{Ab}$ kuhlung in die mit Feuer beheizten "Leuchtolkessel" gepumpt, das Destillat durch fraktionierende Destillation je nach Bedarf in eine Reihe von Fraktionen zerlegt, deren Zahl 
sich nach den tuberaus mannigfaltigen Anforderungen des Abnehmerkreises richtet. Im allgemeinen unterscheidet man $\mathrm{z}$ wischen Petrolather (Gasolin), Leicht-, Mittel- und Schwerbenzin, von denen die letzten drei sich wieder in zablreiche Sorten gliedern wie z. B. Handelsbenzin, Extraktionsbenzin, Grubenlampenbenzin, Waschbenzin, Motorenbenzin, Lackbenzin, Testbenzin usw. Geringere Intervalle als um zwei Stellen der zweiten Dezimale, also $\mathrm{z}$. B. von 0.680 bis 0.700 , oder von 0.710 bis 0.730 werden bei Festsetzung der Fraktionierungsgrenzen wohl nur selten gewählt, obschon die Kunden in Unkenntnis der unendlich verwickelten chemischen Zusammensetzung des Erdoles, manchmal die wunderlichsten Anforderungen stellen. Man bedient sich z. Zt. vorwiegend des von der Berliner Firma C. Heckmann konstruierten, mitGlockenbuden ausgestatteten Rektifikatiunsapparates. Dieser Apparat arbeitet bekanntlich in der Weise, dass die aus dem mit Wasser gekuhl. ten Dephlegmator in den Rektifikator ablaufende Flussigkeit, die aus dem verflussigten Teile der aus dem. Rektifikator in den Dephlegmator strơmenden Kohlenwasserstoffdämpfe besteht, die Glockenboden bedeckt und so. zur Rektifikation der aus dem Destillationskessel in den Rektifikator gelangenden Dämpfe dient. Die nicht im Dephlegmator verflussig- ten Dämpfe strömen zum Kondensator (Kuhler). Der Zufluss des Kuhlwassers zum Dephlegmator wird so geregelt, dass es sich allmählich, entsprechend der Zunahme des Siedepunktes des dem Kondensator entströmenden Destillates immer mehr erwärmt; man kuhlt gegen den Schluss der Destillation mit siedendem Wasser und lässt, um die letzten, noch als Benzin verwertbaren Anteile des Blaseninhaltes tuberzutreiben, das Wasser aus dem Dephlegmator ablaufen, so dass also dann nur mit wenig wirksamer Luftkuhlung gearbeitet wird. Der die Rektifikation beaufsichtigende Arbeiter soll fortlaufend Aufzeichnungen tiber die Zunahme des spez. Gew. des Destillates und der Temperatur des Kuhlwassers im Dephlegmator machen und hat bestimmte Vorschriften zu befolgen hinsichtlich der Regelung der Zufuhr sowohl von Heizdampf wie auch von Kuhlwasser zum Dephlegmator. Bei sachgemässer Beaufsichtigung arbeitet der ${ }^{\mathrm{H}} \mathrm{Heckmann-Apparat}$ sehr befriedigend; seine, immerhin beschränkte, Leistungsfähigkeit lässt folgendes Zahlenbild erkennen:

Ein Heckmannscher Rektifikator mit 30 Glockenbodden lieferte Fraktionen, deren $\mathrm{Zu}$ sammensetzung die bei der Fraktionierung aus dem Englerkolben erhaltenen nachstehenden Zahlen veranschaulichen. (s. Tabelle I)

Tabelle I

\begin{tabular}{|c|c|c|c|c|c|c|c|c|c|c|c|c|c|c|c|c|c|}
\hline \multirow[t]{2}{*}{ Spez. Gen. } & \multirow{2}{*}{$\begin{array}{c}\text { Beginn des } \\
\text { Sledons }\end{array}$} & \multicolumn{14}{|c|}{ Es destillieren Vol. $\%$ bis ${ }^{\circ} \mathrm{C}$ : } & \multirow{2}{*}{\begin{tabular}{|c|}
$\begin{array}{c}\text { Dostlllatlons. } \\
\text { rhokstand }\end{array}$ \\
Vol. $\%$ \\
\end{tabular}} & \multirow{2}{*}{\begin{tabular}{|l|} 
Verlust \\
Vol. $\%$
\end{tabular}} \\
\hline & & $\mid 40$ & $\mid 50$ & 60 & 70 & 80 & 00 & 100 & 110 & 120 & $\mid 180$ & 140 & 150 & 160 & 164 & & \\
\hline $\begin{array}{c}\text { Petrolather } \\
\text { (Gasolin) ca. } 0.660\end{array}$ & 33 & 26 & 68 & 77 & 87 & 9.t & $\theta 2$ & 93 & \begin{tabular}{|l|l} 
& 98.3
\end{tabular} & 93.6 & 94 & 04.5 & 95 & 96 & 08.6 & 1 & 2.5 \\
\hline $\begin{array}{l}\text { Leichtbenzin } \\
\text { ca. } 0.680\end{array}$ & 61 & - & - & - & 45 & 79 & $\theta 1$ & 97 & 98 & - & - & - & - & - & - & 1.2 & 0.8 \\
\hline $\begin{array}{l}\text { Mittelbenzin } \\
\text { ca. } 0.700\end{array}$ & 83 & - & - & - & - & - & 37 & 80 & 84 & 98 & - & - & - & - & - & 1.2 & 0.8 \\
\hline $\begin{array}{l}\text { Schwerbenzin } \\
\text { ca. } 0720\end{array}$ & 102 & - & - & - & $1-$ & - & - & - & 29 & 72 & 91 & 98 & - & - & - & 1.8 & 0.7 \\
\hline
\end{tabular}

Einen weiteren Beitrag zur Charakterisierung der Leistungstlihigkeit dieses Heckmannschen Rektifikators liefert folgendes Zahlenbild: Vier Destillate, die zu vier Zeitpunkten innerhalb einer Sekunde dem Ausfluss des Kondensators entnommen wurden, besassen die durch nachstehende Zahlen veranschaulichte Zu*ammensetzung: (s.Tabelle II)

Die Destillation des entbenzinierten Oeles (vgl. oben) aus den "Leuchtrlkesseln" gestaltet sich folgendermassen: $\mathrm{Da}$ das, wie oben angegeben, mit Hilfe direkt einströmenden
Wasserdampfes der letzten Anteile niedrigsiedender Kohlenwasserstoffe beraubte, $d \mathrm{~h}$. also moglichst weitgehend entbenzinierte $\mathrm{Oel}$ nicht wasserfrei ist, so sorgt man dafur, dass es den "Leuchtrlkesseln" in noch warmem Zustande zugefuhrt wird; das Wasser stellt sich dann bald ab und kann ziemlich vollständig abgezogen werden. Zunłchst wird das Heizfeuer verhaltnismässig schwach gehalten, um einen möglichst grossen Anteil von noch auf Benzin zu verarbeitenden Kohlenwasserstoffen zu erhalten. Wenn das spez. 
Gew. des Destillates auf 0.735 gestiegen ist, beginnt die Leuchtølfraktion. Man gibt stärkeres Feuer und nimmt nun meistens zunächst zwei Fraktionen, eine niedriger siedende
Zersetzungsdestillation, der im Grossbetriebe pennsylvanisches und zum Vergleiche rumbinisches Erdol unterworfen wurde, ergab die halbstundlich erfolgende Bestimmang des

Tabelle II

\begin{tabular}{|c|c|c|c|c|c|c|c|c|c|c|c|c|c|c|c|}
\hline \multirow[t]{2}{*}{$\begin{array}{l}\text { Spaz. Gew. } \\
\text { bol } 15^{\circ}\end{array}$} & \multirow{2}{*}{$\begin{array}{c}\begin{array}{c}\text { Beplan das } \\
\text { Sledens }\end{array} \\
\text { bei }{ }^{\circ} \mathrm{C} \\
\end{array}$} & \multicolumn{12}{|c|}{ Es destillieren Vol. $\%$ bis ${ }^{\circ} \mathrm{C}$ : } & \multirow{2}{*}{\begin{tabular}{|c|}
$\begin{array}{c}\text { Destillatious- } \\
\text { ruckstand }\end{array}$ \\
Vol. $\%$ \\
\end{tabular}} & \multirow{2}{*}{$\frac{\text { Vorlunt }}{\text { Vol. } \%}$} \\
\hline & & 40 & 50 & 60 & 70 & 80 & 80 & 100 & 110 & 120 & 130 & 140 & 150 & & \\
\hline 0.651 & 83 & 21 & 54 & 79 & 95 & 97 & - & - & - & - & - & - & - & 1.1 & 1.9 \\
\hline 0.682 & 47 & - & 1 & 80 & 67 & 81 & 91 & 96 & 98 & - & - & - & - & 1.2 & 0.8 \\
\hline 0.709 & 73 & - & - & - & - & 80 & 69 & 97 & 88.2 & - & - & - & - & 1.2 & 0.6 \\
\hline 0.742 & 106 & - & - & - & - & - & - & - & 9 & 65 & 88 & 96.6 & 98.6 & 1.8 & 0.2 \\
\hline
\end{tabular}

(Fraktionsgrenzen: 0.735 bis 0.765 ) und eine höher siedende (Fraktionsgrenzen 0.765 bis ca. 0.805) ab. Die erstere Fraktion wird im Gemisch mit dem durch Zersetzung erhaltenen Destillate zu geworhnlichem, die letztere zu besserem Leuchtol, sog. Luxuswure, verarbeitet. Nicht selten trennt man noch eine Fraktion mit den Grenzen 0.795 bis ca. 0.805 $a b$, die zu einem Sicherheits-Leuchtol fur besondere Zwecke, z. B. fur Leuchtturme verarbeitet wird. $\mathrm{Ob}$ es zweckmässig ist, bei der Destillation der höher siedenden Anteile, etwa oberhalb der Grenze 0.785 in den Kesselinhalt einen Strom uberhitzten Dampfes einzufuhren, dartuber sind die Ansichten geteilt. Der uberhitzte Dampf soll Zersetzungsvorgänge tunlichst verhliten, er wirkt aber andererseits dadurch ungtunstig, dass er die Fraktionierung beeinträchtigt.

Auf die im Vorstehenden geschilderte Phase der einfachen Fraktionierung folgt die Zersetzungsdestillation, das Kracken (cracking). Sobald das spez. Gew. des Destillates die bezeichnete obere Grenze $(0.805$ oder auch wohl 0.810 ) erreicht hat, wird das Heizfeuer geschwächt, so dass die Destillation zunächst eine Unterbrechung erfährt. Wenn man alsdann wieder vorsichtig mehr Wörme zuftuhrt - die Erfahrung spielt hier den Lehr- spez Gew. des dem Kondensator entstromenden Destillates Zahlenreihen, denen dio nachstehenden Daten entnommen sind (siehe Tab. III).

Beim pennsylvanischen Erdöle also glatte Zersetzung, die zugefuhrte Wurme verrichtet zum grossen Teile chemische Arbeit; beim rumänischen entweder gar keine Zersetzung oder nur in geringem Umfange, die zugofuhrte Wärme verrichtet vorwiegend physikalische Arbeit. Das rumänische Erdöl eignet sich demnach nicht zur Verarbeitung auf Leuchtöl, das durch Zersetzungsdestillation gewonnen wird.

Sobald die Menge des in der Zeiteinheit erzeugten Zersetzungsdestillates wesentlich geringer wird, verstärkt man die Wärmezufuhr und lässt gleichzeitig uberhitzten Dampf in den Kesselinhalt strömen, um den Zersetzungsvorgang moglichst auszuschalten. E. destilliert nun in starkem Laufe ein als ${ }_{n} \mathrm{PQ}$ raffinmasse" bezeichnetes Oel, das als ein Gemisch von Mittelöl und dunnem Lagerschmierol zu bezeichnen ist und, wenn das das Erdరl, wie es beim pennsylvanischen der Fall ist, paraffinreich ist, einen grossen Anteil dieses Paraffing enthält. $\mathrm{Ob}$ es zweckmässig iat, diese "Paraffinmasse" in mohrere Fraktionen zu zerlegen, das hängt von dex

\section{Tabelle III}

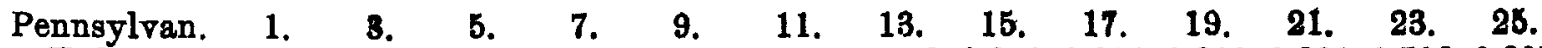

$\begin{array}{lllllllllllllllll}\text { Erdöl: } & 0.805 & 0.805 & 0.804 & 0.802 & 0.807 & 0.802 & 0.803 & 0.801 & 0.804 & 0.800 & 0.801 & 0.798 & 0.805\end{array}$

$\begin{array}{cllllllllllllll}\text { Rumänisch. } & 0.787 & 0.794 & 0.803 & 0.811 & 0.816 & 0.822 & 0.827 & 0.832 & 0.837 & 0.840 & 0.843 & 0.847 & 0.850\end{array}$

meister - so erhält man ein Deslillat, dessen spez. Gew. sich längere Zeit hindurch, d. h. während 40 und mehr Stunden innerhalb der Grenzen 0.800 und 0.810 halten lissst, wie folgende dem Grossbetriebe entstammenden Zahlen zeigen. Bei einer dreissigstundigen
Verwertungsmoglichkeit des Mittel- und Schmieröles ab.

Diese letzte Destillationsphase darf in Interesse einer Schonung des Kesselbodens nicht zu weit getrieben werden. U.m den richtigen Zeitpunkt nicht zu verpassen, kanil 
man sich entweder nach der Menge des erhaltenen Destillates oder nach der Destillationsdauer oder endlich nach gewissen Anzeichen hinsichtlich der Farbe und der Menge des in der Zeiteinheit erhaltenen Destillates richten. Ein erfahrener Destillationsmeister weiss den Punkt genugend genau zu treffen; man verringert die Wärmezufuhr ("lässt das Feuer sacken ${ }^{\text {") }}$ und ermittelt nach einiger Zeit durch Einfuhrung einer graduierten Eisenstange in das Messrohr die Hohe der im Kessel verbliebenen Oelschicht. Dieser Oelruckstand, der, wenn man ihn nicht einfach ablaufen lassen kann, abgezapft und in kleine widerstandsfähige, also ziemlich dickwandige Destillationskessel ubergefuhrt werden muss, hat bei gewöhnlicher Temperatur eine zähflussige Beschaffenheit. Man zapft ihn daher in noch heissem Zustande in die Transportgefüsse $a b$, indessen darf man hinsichtlich seiner Temperatur beim Abzapfen oder Ablaufenlassen eine obere Grenze nicht uberschreiten, da er sich andernfalls in Berthrung mit der Luft entzundet. Man pflegt daher versuchsweise eine gewisse Menge in ein kleineres Gefäss abzulassen, dessen Einrichtung ermoglicht, durch Abschneiden der Luftzufuhr einer schon eingetretenen Entzlindung zu begegnen. Die geleerten Leuchtolkessel lässt man so lange sich abkuhlen, bis die in dieser Hinsicht sehr abgehärteten Reinigungsarbeiter sich in ihnen aufzuhalten vermogen.

(Fortsetzung folgt.)

\section{BERICHTE.}

\section{A. Theorie.}

G. Grasser. Chemische Untersuchung thber die Stone der Birke (Colleginm 1916, S. 445). Durch Aus. ziehen junger, ungetrockneter Birkenblatter mit kaltem Toluol wurde ein hartes, sprodes, braunes Har z gewonnen, nach entsprechender Reinigung zeigto es den Schmelzpunkt $62^{\circ}$ und die Zusammensetzung eines sauren Esters, $\mathrm{C}_{94} \mathrm{H}_{60} \mathrm{O}(\mathrm{COOH})\left(\mathrm{COOC}_{4} \mathrm{H}_{0}\right)$. Beim Verseifen und Ansauern liefert der Ester die freie Dicarbons a ure, $\mathrm{C}_{24} \mathrm{H}_{80} \mathrm{O}(\mathrm{COOH})_{1}$ in Form eines rotgelben, durchsichtigen, spróden Harzes. Der Korper ist wahrscheinlich identisch mit der von Kosmann aus Birkenblattern isolierten Betuloretinsaure, $\mathrm{C}_{26} \mathrm{H}_{66} \mathrm{O}_{\mathrm{B}}$. Bei der Oxydation mit Salpetersäure liefert der Ester ausser der Dicarbonsăure But ters a u re. Das am Kern sitzende funfte Sauerstoffatom gehort woder einer Hydroxyl-, noch einer Aldehyd-, noch einer Ketongruppe an.

Cl. Richardson. Wesen und Ursprung ron Petroleum und Asphalt (Kolloid-Ztschr. 20, 118). Der Verf. ist durch eingehende Forschungen auf obigem Gebiete bekannt. Er stellte fest, dasa die Zusammensetzung des Asphaltes, welcher den berihmten See auf dar Insel Trinidad bildet, an allen Punkten des Sees ausserordentlich gleichmussig ist, or onthalt durchschnittlich $28,0 \%$ Wasser und Gas, $39,0 \%$ Bitumen (18slich in kaltem Schwefelkohlenstof), $0,3 \%$ Bitumen, das von dispersen Mineralbestandteilon adsorbiert und zurtickgehalten wird, 27,2\% Mineralbestandteile und 4,2\% Hydratwasser (des Tons). Dagegen ist die Konsistenz des Asphaltes nicht uberall dieselbe, in der Nahe des Zentrums findet oin andanernder Zufluss von neuem Material statt, das weich und knetbar ist und uberschtissiges Wasser enthslt. Im Lanf der Jahre erhartet es und nimmt die durchschnittliche Konsistenz an. Diese Erhsrtang fuhrt nun Verf. auf die Oberfluchenwirkung zwischen kolloiden Minerslbestandteilen und noch vorhandenem Erd o I zurtick. Fr nimmt an, dass das ans der Tiefo anfsteigende Erdol eino aus Ton, feinem Sand and Wasser bestehonde Schicht durchstreichen muss, mit der $n$ eine innige Emulsion bildet, begtinstigt durch die starke Gamentwickelung infolge der Druckverminderung.
Verf. untersuchte ferner das $\mathrm{Natargas}$, wie os an verschiedenen Stellen des Sees und seiner Umgebung zu Tage tritt. Er fand als Hauptbestandtoil M e than, ferner A eth a $n$, etwas Schwefelwasserstoft und betrachtliche Mengen, bis za $39 \%$ Kohlen. s u re. Verf. kommt za der Anschaunng, dass anch das Erdol aus Erdgas durch die Oberflachenwirkung von Sanden gebildet wird, mit denen es in der Tiefe lange Zeit in Berthrung war. Er nimmt an, dass fur die As phaltbildung der hohe Kohlen. săuregehalt der Erdgase charakteristisch ist, whhrend die normalen Erdole im grossen ganzen aus Methan. Aethan-Mischungen entstehen. Die Verschiedenheit der Roherdole ruhrt von dem verschiedenen Verteilungszustand und Oberfluchencharakter der Sando her. Von Einfluss ist ausser dom Kohlensaure- auch der Schwefelwasserstoffgehalt. Wo Erdgas ohne Erdol vorkommt, muss angenommen werden, dass ersteres nicht gentigend lange Zeit mit dem Sande in Beruhrung oder dass dessen Oberflachenwirkung unzureichend war.

\section{B. Analyse.}

P. Fritzsche. Zur Fettbestimmang in Fleischwaren (Chem.-Ztg. S. 807). 5 Wurst werden mit $10 \mathrm{cc}$ 8-10\% iger alkoholischer Lauge bis zum Verdunsten des Alkohols erwarmt, alsdenn in einem $50 \mathrm{cc}$-Schuttelzylinder mit Salzsture zersetzt und mit Aether ausgeschuttelt. Ein aliquoter Teil der Aetherlosung wird mit Alkohol gemischt und titriert, $1 \mathrm{cc} n /$ Lauge soll 67,5 $\mathrm{mg}$ Fettakure entsprechen, Verf. gibt aber zu, dass diese Zahl wahrscheinlich nur für $\mathrm{Sch}$ we in $\mathrm{e}$ fett richtig sei. (Obige Zahl entspricht einem mittleren Molekulargewicht von 270 und ist daher auch fur Schweinefett noch zu niedrig, Benedikt-Ulzer gibt den Wert 278 an, er kann aber noch höher liegen. Im ubrigen hat Ma y rh ofer schon 1896 dio direlete Behandlnng der Wurstwaren mit alkoholischer Lauge rwecks Bestimmung des Fettes und vor allen Dingen auch des Starkmehls rorgeschlagen. Fa.)

1. 1. Besson. Zur Wasserbestimmung durch DesUllation (Chom.-Ztg, S. 846). Verf. schickt roraus, dase oine Wasserbostimmung mit Ailfo der Destil- 